\title{
Erratum to: MiRNA expression in the cartilage of patients with osteoarthritis
}

\author{
Marta Kopańska 1,2†, Dariusz Szala ${ }^{3 \dagger}$, Joanna Czech ${ }^{1,2}$, Natalia Gabło ${ }^{1,2}$, Krzysztof Gargasz ${ }^{4}$, Mateusz Trzeciak ${ }^{3}$, \\ Izabela Zawlik ${ }^{1,2}$ and Sławomir Snela ${ }^{3^{*}}$
}

\section{Erratum}

In the original publication of this article [1], was an error in the abstract and the methods section which was published with an incorrect kit citation.

The error 1:

Total RNA (containing miRNA species) from cartilage was isolated using a mirVana miRNA Isolation Kit.

Should be read instead:

Total RNA (containing miRNA species) from cartilage was isolated using a miRCURYTM Isolation Kit.

The error 2:

For miRNA profiling, total RNA was purified and prepared. Total RNA (containing miRNA species) from cartilage was isolated using a mirVana miRNA Isolation Kit (Exiqon, Vedbaek, Denmark) following the manufacturer's suggested protocol.

Should be read instead:

For miRNA profiling, total RNA was purified and prepared. Total RNA (containing miRNA species) from cartilage was isolated using a miRCURYTM Isolation Kit (Exiqon, Vedbaek, Denmark) following the manufacturer's suggested protocol.

\footnotetext{
Author details

${ }^{1}$ Laboratory of Molecular Biology, Centre for Innovative Research in Medical and Natural Sciences, University of Rzeszow, Warzywna 1A, 35-959 Rzeszow, Poland. ${ }^{2}$ Department of Genetics, Chair of Molecular Medicine, Faculty of Medicine, University of Rzeszow, Warzywna 1A, 35-959 Rzeszow, Poland. ${ }^{3}$ Clinical Orthopedics and Traumatology Department in Clinical Hospital 2, 35-301 Rzeszow, Poland. ${ }^{4}$ Data Analysis Laboratory, Centre for Innovative Research in Medical and Natural Sciences, Faculty of Medicine, University of Rzeszow, Rzeszow, Poland.
}

Received: 8 May 2017 Accepted: 8 May 2017

Published online: 13 June 2017

\section{Reference}

1. MiRNA expression in the cartilage of patients with osteoarthriti. J Orthop Surg Res. 2017;12:51. doi: 10.1186/s13018-017-0542-y

\footnotetext{
* Correspondence: ssnela@poczta.onet.pl

${ }^{\dagger}$ Equal contributors

${ }^{3}$ Clinical Orthopedics and Traumatology Department in Clinical Hospital 2,

35-301 Rzeszow, Poland

Full list of author information is available at the end of the article
} 